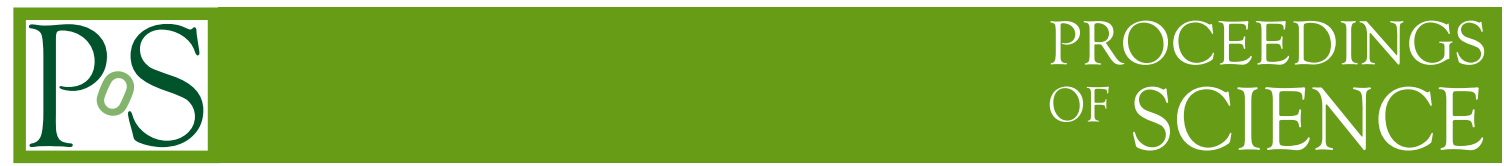

\title{
Application of parton branching TMDs to Z-boson production
}

\author{
Jindrich Lidrych, ${ }^{a, *}$ Patrick L.S. Connor, ${ }^{a}$ Francesco Hautmann ${ }^{b, c}$ and Hannes Jung ${ }^{a}$ \\ ${ }^{a}$ Deutsches Elektronen-Synchrotron, \\ Notkestrasse 85, 22607 Hamburg, Germany \\ ${ }^{b} R A L$ and University of Oxford, \\ OX1 3PU, United Kingdom. \\ ${ }^{c}$ Elementaire Deeltjes Fysica, Universiteit Antwerpen, \\ 2020 Antwerpen, Belgium \\ E-mail: jindrich.lidrych@desy.de, patrick.connor@desy.de, \\ hautmann@thphys.ox.ac.uk, hannes.jung@desy.de
}

Transverse Momentum Dependent (TMD) parton distribution functions obtained within the Parton Branching (PB) approach offer a wide spectrum of applications to describe processes in pp as well as in ep interactions. In this proceedings we show an example of application of the PB TMD method to describe Z-boson production in high energy pp interactions. The PB TMD approach together with dedicated parton showers provide a new and consistent way to describe not only inclusive but also multi-jet distributions at different center-of-mass energies.

40th International Conference on High Energy physics - ICHEP2020

July 28 - August 6, 2020

Prague, Czech Republic (virtual meeting)

\footnotetext{
${ }^{*}$ Speaker
} 


\section{Introduction}

The transverse momentum dependent parton distribution functions (TMD PDFs) play an important role in the description of small transverse momentum physics as well as small $x$ physics. In order to obtain TMD PDFs in a wider kinematic range, we can employ the parton branching (PB) method to numerically solve TMD evolution equations [1]. The PB method is an iterative procedure using the concept of resolvable and non-resolvable branching and applying Sudakov form factors to describe the probability for no resolvable branchings from one evolution scale to another. In each step, the kinematics of the splitting are known. This fact enables us to extract the TMDs. More detailed description of the PB method can found in [2].

In this proceedings, we present an application of PB-TMDs to LHC physics. In particular, the TMDs (PB-NLO-2018-Set2) obtained from the fit [3] to inclusive deep-inelastic scattering data from HERA are used to make next-to-leading-order (NLO) TMD theoretical predictions for the transverse momentum spectrum of Z-bosons at LHC energies [4].

\section{Production of Z-bosons at the $\mathrm{LHC}$ energies}

The measurement of Z-bosons production can serve as a precision test of the Standard model. Nowadays measurements of Z-bosons with the lepton pair in the final state are very precise and the current experimental uncertainties in the low transverse momentum region of Z-bosons are dominated by uncertainty related to the integrated luminosity [5].

In order to describe the measurements at low transverse momenta $\left(p_{\mathrm{T}}<O\left(m_{\mathrm{Z}}\right)\right)$, soft-gluon perturbative resummations and nonperturbative contributions need to be taken into account. The PB method [1,2] incorporates both, by defining nonperturbative TMDs (to be fitted to data) and evaluating their evolution through perturbative kernels, given in terms of Sudakov form factors and angular-ordered phase space. In Figure 1 (left), the predictions for the transverse momentum spectrum of Z-bosons obtained with PB-TMD [4] are compared with the ATLAS measurements at $\sqrt{s}=8 \mathrm{TeV}$ [6]. The matrix element for inclusive Z-boson production is calculated at NLO using MC@NLO [7]. The predictions are shown together with the corresponding uncertainties coming from the TMD densities and the independent variation of the renormalization and factorization scale $\left(\mu_{\mathrm{F}}\right.$ and $\left.\mu_{\mathrm{R}}\right)$. The prediction based on PB-TMDs agrees reasonably well with the data at low transverse momenta. The disagreement at the higher transverse momenta is related to the missing higher order jet multiplicity in the matrix element. In the recent measurement at $\sqrt{s}=13 \mathrm{TeV}$ reported by the CMS Collaboration [5], various resummed calculations like GENEVA [8] and RESBOS [9] were utilized in the comparison with data. Figure 1 (right) shows that PB-TMD method provides a competitive prediction, which agrees with the data at the low transverse momenta similarly well as GENEVA and RESBOS models.

\section{Conclusion}

In this proceedings, we briefly introduced the PB-TMDs and highlighted the application of parton branching method to the Z-boson production at the LHC energies. Good agreement between PB-TMD predictions and low transverse momentum data was found. 

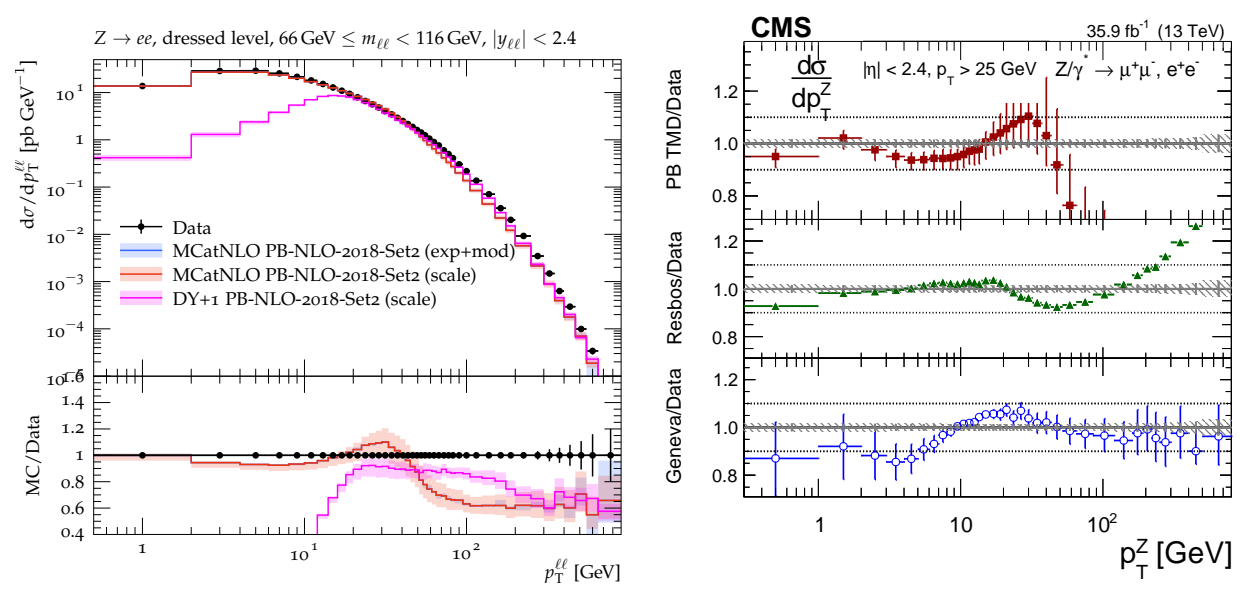

Figure 1: Transverse momentum $p_{\mathrm{T}}$ spectrum of Z-bosons measured at the LHC. The left plot shows comparisons of the measurement from $\sqrt{s}=8 \mathrm{TeV}$ [6] to predictions [4] from MC@NLO with PB-TMD, including uncertainties from the TMDs and scale variation. The right plot shows the latest results from $\sqrt{s}=13 \mathrm{TeV}$ measured by the CMS and compared to various MC predictions. Taken from [5].

\section{References}

[1] F. Hautmann, H. Jung, A. Lelek, V. Radescu and R. Zlebcik, Soft-gluon resolution scale in QCD evolution equations, Phys. Lett. B 772 (2017) 446 [1704 . 01757].

[2] F. Hautmann, H. Jung, A. Lelek, V. Radescu and R. Zlebcik, Collinear and TMD Quark and Gluon Densities from Parton Branching Solution of QCD Evolution Equations, JHEP 01 (2018) 070 [1708.03279].

[3] A. Bermudez Martinez et al., Collinear and TMD parton densities from fits to precision DIS measurements in the parton branching method, Phys. Rev. D 99 (2019) 074008 [1804 . 11152].

[4] A. Bermudez Martinez et al., Production of Z-bosons in the parton branching method, Phys. Rev. D 100 (2019) 074027 [1906.00919].

[5] CMS collaboration, Measurements of differential Z boson production cross sections in proton-proton collisions at $\sqrt{s}=13 \mathrm{TeV}$, JHEP 12 (2019) 061 [1909.04133].

[6] ATLAS collaboration, Measurement of the transverse momentum and $\phi_{\eta}^{*}$ distributions of Drell-Yan lepton pairs in proton-proton collisions at $\sqrt{s}=8 \mathrm{TeV}$ with the ATLAS detector, Eur. Phys. J. C 76 (2016) 291 [1512.02192].

[7] J. Alwall et al., The automated computation of tree-level and next-to-leading order differential cross sections, and their matching to parton showers, JHEP 07 (2014) 079 [1405.0301].

[8] S. Alioli, C.W. Bauer, C. Berggren, F.J. Tackmann and J.R. Walsh, Drell-Yan production at NNLL'+NNLO matched to parton showers, Phys. Rev. D 92 (2015) 094020 [1508.01475].

[9] C. Balazs and C. Yuan, Soft gluon effects on lepton pairs at hadron colliders, Phys. Rev. D 56 (1997) 5558 [hep-ph/9704258]. 